\title{
Controlling nutritional status score predicts postoperative complications after hip fracture surgery
}

Toshio Yagi ${ }^{1}$, Yusuke Oshita ${ }^{2}$, Ichiro Okano ${ }^{1,3^{*}}$ D, Takuma Kuroda ${ }^{1}$, Koji Ishikawa' ${ }^{1}$, Takashi Nagai ${ }^{1}$ and Katsunori Inagaki ${ }^{1}$

\begin{abstract}
Background: Controlling Nutritional Status (CONUT) score is calculated using laboratory values, including serum albumin, total cholesterol concentration, and total lymphocyte count; it is reportedly valuable for making nutritional assessments. One advantage of CONUT score over other nutritional assessments is that it can be calculated retrospectively using only objective laboratory values. Studies demonstrated that CONUT score was a useful tool for predicting prognosis and complications in various surgical conditions. Nevertheless, few studies utilized the score as a potential predictive marker for postoperative complications among hip fracture patients. The purpose of this study was to determine the association between CONUT score and postoperative complications in hip fracture patients.
\end{abstract}

Methods: We retrospectively reviewed 211 elderly patients who underwent hip fracture surgery at a single institution from 2013 to 2018. CONUT score was calculated using preoperative routine laboratory tests for serum albumin, total cholesterol concentration, and total lymphocyte count. As potential confounders, we extracted data such as patient age, sex, fracture type, and general conditions/comorbidities, as defined by the American Society of Anesthesiologists Physical Status (ASA-PS) classification and the Charlson Comorbidity Index (CCI). Postoperative complications were defined as a Clavien-Dindo classification of 1 or more. Simple and multivaribale logistic regression analyses were performed to assess the incidence of postoperative complications as the outcome measures.

Results: The mean age [IQR] was 86 [80-90], and $80.1 \%$ of the reviewed patients were female. Based on the CONUT scores, $78.7 \%$ of hip fracture patients were classified as malnourished; $18 \%$ experienced postoperative complications. Simple analyses revealed significant risk factors for postoperative complications, including age, the ASA-PS, the CCI, and the CONUT score. Multivariable analysis found that CONUT score was the independent risk factor for postoperative complications (odd ratio $=1.21,95 \%$ confidence interval $=1.01-1.45, p=0.04$ ).

Conclusions: Preoperative CONUT scores are independently associated with the incidence of postoperative complications. CONUT score can be used for risk assessment in hip fracture patients to predict early postoperative complications.

Keywords: Hip fracture, Nutrition, Controlling nutritional status, CONUT score, Postoperative complication, Elderly patient

\footnotetext{
* Correspondence: ichiro.okano.e31@gmail.com

'Department of Orthopedic Surgery, Showa University School of Medicine,

1-5-8 Hatanodai Shinagawa-ku, Tokyo 142-8555, Japan

${ }^{3}$ Department of Orthopedic Surgery, Ohta-Nisihinouchi Hospital, 2-5-20

Nishinouchi, Koriyama 963-8558, Japan

Full list of author information is available at the end of the article
}

(c) The Author(s). 2020 Open Access This article is licensed under a Creative Commons Attribution 4.0 International License, which permits use, sharing, adaptation, distribution and reproduction in any medium or format, as long as you give appropriate credit to the original author(s) and the source, provide a link to the Creative Commons licence, and indicate if changes were made. The images or other third party material in this article are included in the article's Creative Commons licence, unless indicated otherwise in a credit line to the material. If material is not included in the article's Creative Commons licence and your intended use is not permitted by statutory regulation or exceeds the permitted use, you will need to obtain permission directly from the copyright holder. To view a copy of this licence, visit http://creativecommons.org/licenses/by/4.0/ The Creative Commons Public Domain Dedication waiver (http://creativecommons.org/publicdomain/zero/1.0/) applies to the data made available in this article, unless otherwise stated in a credit line to the data. 


\section{Background}

Currently osteoporosis is one of the most prevalent diseases in many developed countries because of an aging population. The number of patients with osteoporotic fractures is expected to increase rapidly [1, 2]. Osteoporotic hip fracture has the greatest impacts on patients' function and life expectancy among all fragility fractures. Elderly patients who sustain a hip fracture are more likely to sustain postoperative medical and surgical complications such as pneumonia, heart failure, and urinary tract infections than other age groups of surgical patients [3-5].

Poor nutritional status is one of the major contributing factors for postoperative complications in many types of surgery [6, 7]. Several nutritional assessment systems have been introduced. The Subjective Global Assessment (SGA) enables the clinician to determine the nutritional status of the patient; it has been widely used as a global assessment of nutritional status for various surgical conditions [8]. However, one major drawback of these assessment systems is that they include one or more measurements that inherently depend on a degree of subjectivity. Another major drawback is that these subjective measurements cannot be conducted retrospectively. In other words, if one of these assessments is not conducted prior to surgery, it is impossible to evaluate later on the surgical risk associated with nutritional status with these assessment systems.

Controlling Nutritional Status (CONUT) was first introduced by Ignacio et al. [9]; it requires only common laboratory tests that are often performed routinely in presurgical assessments and/or periodical health assessment examination by general practitioners. CONUT allows examiner-independent and retrospective evaluations for nutritional status. Previous studies demonstrated that CONUT highly correlated with SGA [10] and was useful to stratify the risk for postoperative complication among patients undergoing surgery for gastrointestinal and hepatopancreatobiliary cancers [11]. Despite these benefits of CONUT, few studies have addressed osteoporotic hip fractures. Therefore, the purpose of this study was to investigate the association between nutritional status evaluated using CONUT and postoperative complications.

\section{Methods}

\section{Study population}

Institutional ethical committee approval was obtained for this study (No10-10). We retrospectively reviewed clinical data of consecutive patients with hip fracture between 2013 and 2018 at a single community-based hospital. We excluded young patients $(<50 \mathrm{y})$, those receiving conservative treatment, and those who suffered high-energy trauma or pathological fracture. We recorded potential confounders, including age, sex, fracture type, blood hemoglobin concentration, days until return to long-term residence, discharge destination, American Society of Anesthesiologists physical status (ASA-PS) classification, and Charlson Comorbidity Index (CCI) [12].

\section{CONUT score and complication assessment}

Preoperative blood samples were taken on the day of admission or the following day in patients admitted outside of daily working hours. The preoperative CONUT scores were calculated using the results of three laboratory tests; serum albumin concertation, lymphocyte count, and total cholesterol concentration (Table 1).

As the primary outcome measure, postoperative complications were documented utilizing the Clavien-Dindo (CD) classification for surgical complications [13]. The $\mathrm{CD}$ classification is a five-grade assessment system for general postoperative complications; Grade I is defined by deviation from the normal postoperative course, including noninfectious diarrhea, or transient elevation of serum creatinine. Grade II is defined by conditions requiring medical therapy without surgical treatment, including pneumonia treated with antibiotics in the ward, or urinary tract infection requiring antibiotics. Grade III is defined by surgical, endoscopic or radiological intervention, including wound infection requiring debridement, or bradyarrhythmia requiring pacemaker implantation under local anesthesia. Grade IV is defined by life-threatening complications requiring intensive care, including respiratory failure requiring intubation, or ischemic stroke. Grade V is defined by death due to complications. We defined a postoperative complication as any type of postoperative complication with $\mathrm{CD}$ grade $\geq 1$ occurring between the time of surgery until 30 days after the primary procedure.

\section{Statistical analysis}

Statistical analyses were performed utilizing the Chisquared test for categorical variable comparisons, and logistic regression test for simple and multivariable analyses. The statistical significance was set at $p<0.05$. All

Table 1 Assessment of the nutritional status using CONUT

\begin{tabular}{lllll}
\hline Parameter & \multicolumn{4}{l}{ Malnutrition status } \\
\cline { 2 - 5 } & None & Light & Moderate & Severe \\
\hline Serum albumin $(\mathrm{g} / \mathrm{dL})$ & $\geqq 3.5$ & $3.0-3.49$ & $2.5-2.99$ & $<2.5$ \\
Albumin score & $\mathbf{0}$ & $\mathbf{2}$ & $\mathbf{4}$ & $\mathbf{6}$ \\
$\begin{array}{l}\text { Total lymphocyte count } \\
\left(/ \mathrm{mm}^{3}\right)\end{array}$ & $\geqq 1600$ & $1200-1599$ & $800-1199$ & $<800$ \\
Lymphocyte score & $\mathbf{0}$ & $\mathbf{1}$ & $\mathbf{2}$ & $\mathbf{3}$ \\
Total cholesterol $(\mathrm{mg} / \mathrm{dl})$ & $\geqq 180$ & $140-179$ & $100-139$ & $<100$ \\
Cholesterol score & $\mathbf{0}$ & $\mathbf{1}$ & $\mathbf{2}$ & $\mathbf{3}$ \\
Total score & $\mathbf{0 - 1}$ & $\mathbf{2 - 4}$ & $\mathbf{5 - 8}$ & $\mathbf{9 - 1 2}$ \\
\hline
\end{tabular}

CONUT Controlling Nutritional Status 
the analyses were carried out using $\mathrm{R}$ software ( $\mathrm{R}$ for 3.4.1 GUI 1.64) [14].

\section{Results}

Patient demographics and clinical characteristics

A total of 211 patients were identified (Fig. 1). The median age [IQR] was 86 [ $80-90$ ] years; $80.1 \%$ of the patients were female; $66.8 \%$ of patients were ASA-PS class 2 , and the median CCI score [IQR] was 1.0 [0-2]; 78.7\% of the patients were malnourished, as defined by a CONUT score $>1$. Of these 211 patients, 39 (18.5\%) experienced at least one postoperative complication (14 urinary tract infections, six heart failures, four surgical site infections, four re-fractures, three pneumonias, three arrhythmias, three cases of enteritis, and 10 others) (Tables 2 and 3). The mean duration between the hospital admission to final discharge to long-term residences, such as home or a nursing care facility, was 29.5 [21-41] days in patients with no postoperative complications and 43 [31-52.5] days in patients with postoperative complications $(p=0.03)$. Thirty-day mortality rates were $0.95 \%$. The percentage of patients originally living at home who were discharged home was $38.4 \%$. Demographics and clinical characteristics of the patients are summarized in Table 4.

\section{Risk factors for postoperative complications}

To identify potential risk factors for postoperative complications, simple analyses were carried out. Preoperative age $(p=0.01)$, ASA-PS $(p=0.02)$, Hemoglobin $(p=$ $0.004)$ and CONUT score $(p=0.002)$ were significant risk factors for postoperative complications (Table 4).
Multivariable logistic regression analysis, adjusted for age, ASA-PS and CCI, demonstrated that CONUT score was the independent risk factor for postoperative complication $(\mathrm{OR}=1.21,95 \% \mathrm{CI}=1.01-1.45, p=0.04)$ (Table 5$)$.

\section{Discussion}

We found that the CONUT score was an independent factor for postoperative complications in our population. Of 211 patients, 39 (18\%) had postoperative complications, consistent with results of other studies reporting $19-20 \%$ overall complication rates $[3,4]$.

CONUT was developed by Ignacio de Uli'Barri et al. in 2005 as a screening tool for malnutrition among general hospitalized patients [9]. A recent systematic review and meta-analysis showed that preoperative CONUT score was associated with increased risk of mortality and complications in surgical patients with gastrointestinal and hepatopancreatobiliary cancers [11]. Another retrospective study found that CONUT score was useful for predicting long-term mortality in hospitalized patients with heart failure [15]. Despite abundant evidence showing the usefulness of CONUT score as the index of malnutrition for short and long-term clinical outcomes of various conditions, we found only one study which utilized CONUT score as a predictive marker for hip fracture patients. The results showed that CONUT score predicted the 180-day mortality well in hip fracture patients: the survivors' mean CONUT score was $4 \pm 2$, whereas the non-survivors' CONUT score was $6 \pm 3$ [16]. The study did not include the association between CONUT and postoperative complications, whereas we

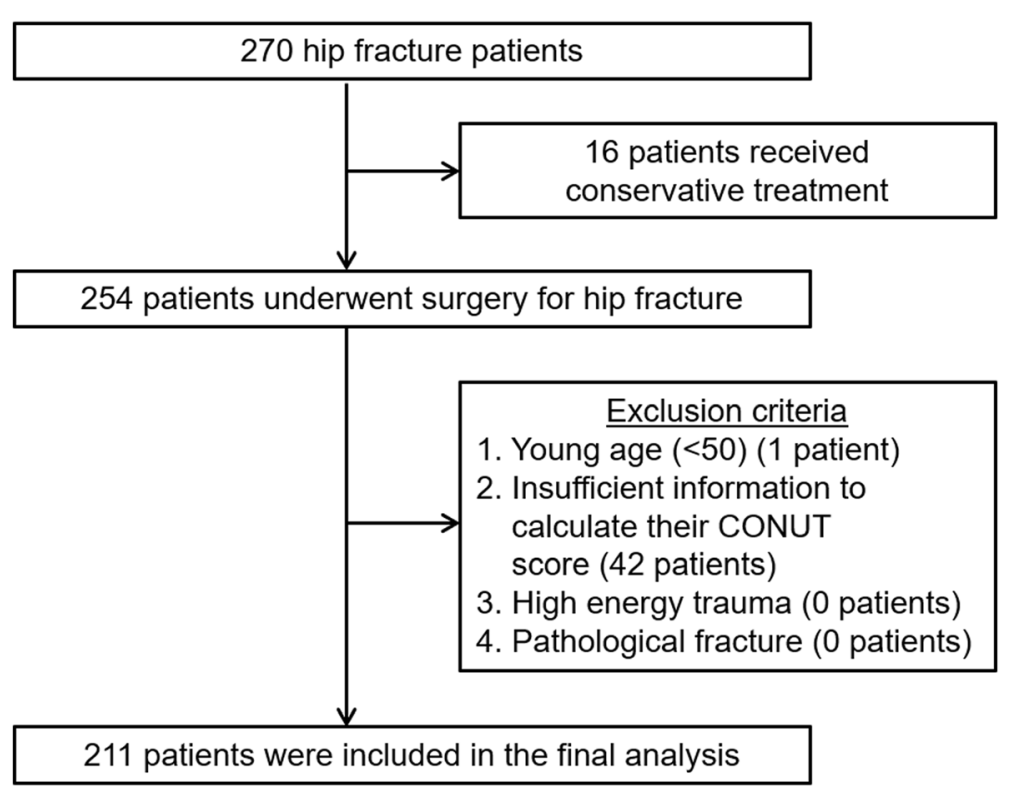

Fig. 1 Flow diagram of inclusion criteria 
Table 2 Postoperative complications

\begin{tabular}{ll}
\hline Postoperative Complication (\%) & \\
\hline Urinary tract infection & $14(29)$ \\
Heart failure & $6(12)$ \\
Surgical site infection & $4(8)$ \\
Re-fracture & $4(8)$ \\
Pneumonia & $3(6)$ \\
Arrhythmia & $3(6)$ \\
Enteritis & $3(6)$ \\
Liver dysfunction & $2(4)$ \\
Implant failure & $2(4)$ \\
Acute myocardial infraction & $1(2)$ \\
Venous thromboembolism & $1(2)$ \\
Asthma & $1(2)$ \\
Gastrointestinal bleeding & $1(2)$ \\
Heterotopic ossification & $1(2)$ \\
Pseudo-gout & $1(2)$ \\
Renal dysfunction & $1(2)$ \\
Cholangitis & $1(2)$ \\
\hline &
\end{tabular}

provided novel information about the association between CONUT score and postoperative complications.

There have been several retrospective studies investigating associations between malnutrition and clinical outcomes in hip fracture patients. These studies utilized serum albumin concentration as the index of malnutrition and showed significant associations between hypoalbuminemia (albumin $<3.5 \mathrm{~g} / \mathrm{dL}$ ) and increased postoperative complications and mortality [17-19]. Another study found that the group with low albumin and low lymphocyte counts at the time of hospital admission had a higher 12-month mortality rate than did the group with a normal albumin concentration [20].

Other studies utilized clinical assessments based on physical examination and post medical history, in which certain elements were associated with malnutrition, as prognostic markers for hip fracture patients. Ahman

Table 3 Clavien-Dindo classification grade

\begin{tabular}{ll}
\hline Clavien-Dindo classification grade (\%) & \\
II & $2(4.7)$ \\
III & $23(53.5)$ \\
IV & $15(34.9)$ \\
V & $1(2.3)$ \\
\hline
\end{tabular}

Grade I is defined by deviation from the normal postoperative course Grade II is defined by conditions requiring medical therapy without surgical treatment Grade III is defined by surgical, endoscopic or radiological intervention

Grade IV is defined by life-threatening complications requiring intensive care Grade $V$ is defined by death due to complications et al. reported that ASA-PS, a system for assessing the fitness of patients for surgery, was an independent predictor of one-year mortality after hip surgery [21]. Another study demonstrated that CCI, based on the number of index comorbidities, correlated with shortand long-term mortality rates after surgical treatment of hip fracture [22]. In our study, only CONUT score showed statistical significance in the multivariate analysis, whereas ASA-PS and CCI were not independent risk factors for complications after hip fracture. One drawback of ASA-PS is that this system inherently depends on the subjectivity of examiners. One study showed that the interrater reliability of ASA-PS was only moderate, with kappa values around 0.60 [23]. ASA-PS includes six categories; however, categories 5 (moribund) and 6 (organ donor) are generally not used for fracture patients, and category 1 (healthy) patients are rare among patients with fragility hip fractures. For these reasons, in real patients with hip fractures, only three categories can be counted. This could be another shortcoming of ASA-PS for detailed risk stratification of hip fracture patients. CCI has less room for subjectivity, because it is based on the number of comorbidities. Nevertheless, one major drawback of CCI is that the severity of each disease is only included in a limited number of items, including diabetes, liver disease, and cancer. For example, heart failure is always allotted one point, regardless of its severity. These points might have contributed to our results and might highlight the importance of objective quantitative nutritional assessment for prediction of postoperative complications after hip fracture surgery.

Compared with other nutritional specific assessments, including SGA, Mini Nutritional Assessment-Short Form (MNA-SF) [24], and Malnutrition Screening Tool (MST) [25], CONUT has unique advantages. First, CONUT score can be calculated only with objective laboratory values. This means that CONUT does not require detailed history-taking. All other nutritional assessments include factors based on past medical history or recent food intake. Hip fracture patients often have dementia [26], making it extremely difficult to collect accurate information using medical interviews. Furthermore, CONUT scores can be retrospectively calculated when needed without physical examinations. This feature is especially useful in the research setting or when treating referred patients. By contrast, it is impossible to calculate the SGA score when certain examinations are not conducted at the time of admission. It was reported that the SGA had a low inter-rater reliability (13\%), despite the fact that kappa increased as examiners gained experience [27]. By contrast, CONUT score is automatically calculated using objective values and is completely free from rater reliability issues. Regarding other measurement systems, Bel et al. reported that the 
Table 4 Demographics, patient characteristics and the results of simple logistic regression analysis

\begin{tabular}{|c|c|c|c|c|c|}
\hline \multirow[t]{2}{*}{ Demographic characteristics } & \multirow[b]{2}{*}{$\begin{array}{l}\text { All patients } \\
(N=211)\end{array}$} & \multicolumn{2}{|c|}{ Postoperative complications } & \multirow[b]{2}{*}{$\underline{\mathrm{OR}(95 \% \mathrm{Cl})}$} & \multirow[b]{2}{*}{$\underline{p \text {-value }}$} \\
\hline & & No $(N=172)$ & Yes $(N=39)$ & & \\
\hline Age (Med [IQR]) & 86 [80-90] & 85 [78.8-90] & 88 [85.5-91.5] & $1.06(1.01-1.11)$ & 0.01 \\
\hline \multicolumn{6}{|l|}{ Sex (\%) } \\
\hline Women & $169(80.1)$ & $137(79.7)$ & $32(82.1)$ & ref & Ref \\
\hline Men & $42(19.9)$ & $35(20.3)$ & $7(17.9)$ & $0.86(0.35-2.1)$ & 0.74 \\
\hline \multicolumn{6}{|l|}{ Fracture type (\%) } \\
\hline Intra-articular & $84(39.8)$ & $68(39.5)$ & $16(41.0)$ & ref & Ref \\
\hline Extra-articular & $122(57.8)$ & $101(58.7)$ & $21(53.8)$ & $0.88(0.43-1.81)$ & 0.73 \\
\hline Subtrochanteric & $5(2.4)$ & $3(1.7)$ & $2(5.1)$ & $2.83(0.44-18.4)$ & 0.28 \\
\hline $\begin{array}{l}\text { Days until return to long-term } \\
\text { residence (Med [IQR]) }\end{array}$ & $31[23-43]$ & $29.5[21-41]$ & $43[31-52.5]$ & $1.02(1.00-1.04)$ & 0.03 \\
\hline \multicolumn{6}{|l|}{ Discharge destination (\%) } \\
\hline Home & $85(40.2)$ & $76(89.4)$ & $9(10.6)$ & ref & Ref \\
\hline Nursing care institution & $126(59.7)$ & $96(76.2)$ & $30(23.8)$ & $2.4(1.16-4.92)$ & 0.02 \\
\hline \multicolumn{6}{|l|}{ ASA class (\%) } \\
\hline 1 & $11(5.2)$ & $11(6.4)$ & 0 & ref & Ref \\
\hline 2 & $141(66.8)$ & $119(69.2)$ & $22(56.4)$ & (PS1-2) & \\
\hline 3 & $58(27.5)$ & $42(24.4)$ & $16(41.0)$ & $2.39(1.16-4.92)$ & 0.02 \\
\hline 4 & $1(0.5)$ & $00.0)$ & $1(2.6)$ & (PS3-4) & \\
\hline CCl (\%) & $1.0[0-2]$ & $1.0[0-2]$ & $1.0[1-2]$ & & \\
\hline 0 & $61(28.9)$ & $57(33.1)$ & $4(10.3)$ & ref & Ref \\
\hline 1 & $71(33.6)$ & $49(28.5)$ & $22(56.4)$ & $6.4(2.06-19.8)$ & 0.01 \\
\hline $2<$ & $79(37.4)$ & $66(38.4)$ & $13(33.3)$ & $2.81(0.86-9.09)$ & 0.09 \\
\hline Hemoglobin (g/dl) (Mean (SD)) & $11.8(1.9)$ & $11.0(1.8)$ & $12.0(1.8)$ & $0.75(0.62-0.91)$ & 0.004 \\
\hline CONUT (med [IQR]) & $3.1[0.00-11.00]$ & $3.0[0.00-11.00]$ & $4.00[0.00-10.00]$ & $1.30(1.10-1.54)$ & 0.002 \\
\hline Normal & $45(21.3)$ & $33(73.3)$ & $12(26.7)$ & & \\
\hline Light & $115(54.5)$ & $91(79.1)$ & $24(20.9)$ & & \\
\hline Moderate & $49(23.2)$ & $36(73.5)$ & $13(26.5)$ & & \\
\hline Severe & $2(0.9)$ & 0 & $2(100)$ & & \\
\hline
\end{tabular}

\section{OR Odds Ratio}

Cl Confidence Interval

$I Q R$ Interquartile Range

ref Reference

ASA-PS American Society of Anesthesiologists Physical Status

$\mathrm{CCl}$ Charlson Comorbidity Index

SD Standard Deviation

CONUT Controlling Nutritional Status

Table 5 Multivariable logistic regression analysis

\begin{tabular}{llll}
\hline Parameters & \multicolumn{3}{l}{ Multivariable Logistic Regression } \\
\cline { 2 - 4 } & OR & $95 \% \mathrm{Cl}$ & -value \\
\hline Age & 1.05 & $0.997-1.10$ & 0.067 \\
ASA-PS & 1.94 & $0.903-4.15$ & 0.089 \\
CCI & 1.13 & $0.822-1.56$ & 0.445 \\
CONUT score & 1.21 & $1.01-1.45$ & $\mathbf{0 . 0 4 0}$
\end{tabular}

Independent variables: Age, ASA-PS, CCl, CONUT score

Dependent variable: Postoperative complication

$\mathrm{Cl}$ Confidence interval

ASA-PS American Society of Anesthesiologists Physical Status

$\mathrm{CCl}$ Charlson Comorbidity Index

CONUT Controlling Nutritional Status specificity of MNA-SF for malnutrition defined by ICD10 -code was not high (49\%). MST demonstrated low sensitivity (60\%) for malnutrition in hip fracture patients [28]. By contrast, CONUT score showed excellent diagnostic ability with area under the curve as 0.86 for clinically diagnosed malnutrition [10]. In certain conditions, CONUT score should be interpreted with caution, as it may be affected by nutritional status, immune status, inflammation, metabolic diseases, and dehydration. For example, in patients with acute bacteremia or certain hematological conditions, the lymphocyte count may be 
disproportionally low. In such cases, a high CONUT score does not necessarily indicate malnutrition.

The present study has some limitations. First, this was a retrospective study conducted at a single institution, and the number of cases was limited. Additionally, because of the retrospective nature of this study and the lack of specific protocols to detect the complications, the incidences of certain complications, such as delirium, were likely underestimated and/or not well-differentiated from other complications. One report demonstrated that delirium was misdiagnosed in over $60 \%$ of cases if the diagnosis was made solely by physicians' impression [29]. To address the accurate estimation of complication rates, standardized diagnostic protocols are warranted in future studies. We investigated the associations between CONUT score and postoperative complications, but did not compare CONUT score to postoperative mobility status or rehabilitation outcome, due to the data availability and our study population including only surgically treated patients. Thus, the results cannot be directly applied to patients undergoing conservative treatment. Furthermore, blood samples were taken at time of admission or the next day; therefore, blood sampling conditions were not consistent, and the performance status was only evaluated with ASA-PS, which is relatively simple. Fourth, there were no direct comparisons with other nutritional indicators such as SGA, MNA-SF, or MST, because of the retrospective nature of this study. This should be addressed in future prospective studies. Additionally, we evaluated complications at 30 days postoperatively, making it impossible to draw any conclusions about long-term prognosis. Studies with longer follow-up are warranted to address this issue. Finally, this study was observational and did not include any results of interventions for malnutrition that might improve the prognosis of hip fracture patient population. Espauella et al. showed that hip fracture patients who received nutritional supplementation had lower complications and hospital length of stay [30]. Another multicenter prospective cohort study showed that abdominal surgical patients who received nutritional support had a lower complication rate than did the control group (25.6\% versus $50.6 \%)$ [31]. In future studies, nutritional interventions based on CONUT assessments should be evaluated to improve the outcomes.

\section{Conclusions}

The CONUT score was an independent predictor of postoperative complications after adjustment for ASAPS and CCI. These findings suggest that the preoperative CONUT score is a useful screening tool for nutritional assessment hip fracture patients to predict early postoperative complications. CONUT can be a useful tool for nutritional assessment in future hip fracture studies and daily practice.

\section{Abbreviations}

CONUT: Controlling Nutritional Status; SGA: Subjective Global Assessment; ASA-PS: American Society of Anesthesiologists physical status; CCI: Charlson Comorbidity Index; CD: Clavien-Dindo; SD: Standard deviation; IQR: Interquartile Range; OR: Odds ratio; Cl: Confidence interval; ref:: Reference; MNA-SF: Mini Nutritional Assessment-Short Form; MST: Malnutrition Screening Tool; ICD: International Statistical Classification of Diseases and Related Health Problems

\section{Acknowledgments \\ Not applicable.}

Authors' contributions

TY conceptualized, collected, and interpreted the clinical data, and wrote the manuscript. YO conceptualized, interpreted the clinical data, and revised the manuscript critically for important content. IO, TK, KI, TN and KI interpreted the clinical data, and revised the manuscript critically for important content. All authors read and approved the final manuscript.

\section{Funding}

The authors received no financial support for the research, authorship, or publication of this article.

Availability of data and materials

Data that support the findings of this study are available from the corresponding author on reasonable request.

Ethics approval and consent to participate

The study was approved by the institutional ethics committee of the Yamanashi Red Cross Hospital approval number: (No10-10).

Consent for publication

Not applicable.

\section{Competing interests}

The authors declare that they have no competing interests.

\section{Author details}

'Department of Orthopedic Surgery, Showa University School of Medicine, 1-5-8 Hatanodai Shinagawa-ku, Tokyo 142-8555, Japan. ${ }^{2}$ Department of Orthopedics, Showa University Northern Yokohama Hospital, 35-1

Chigasaki-cho, Tsuzuki-ku, Yokohama 224-8503, Japan. ${ }^{3}$ Department of Orthopedic Surgery, Ohta-Nisihinouchi Hospital, 2-5-20 Nishinouchi, Koriyama 963-8558, Japan

Received: 10 March 2020 Accepted: 8 July 2020

Published online: 13 July 2020

\section{References}

1. Cooper C, Campion G, Melton L 3 rd. Hip fractures in the elderly: a worldwide projection. Osteoporos Int. 1992;2:285-9.

2. Johnell $\mathrm{O}$. The socioeconomic burden of fractures: today and in the $21 \mathrm{st}$ century. Am J Med. 1997:103:20S-5S discussion 25S-6S.

3. Roche JJ, Wenn RT, Sahota O, Moran CG. Effect of comorbidities and postoperative complications on mortality after hip fracture in elderly people: prospective observational cohort study. BMJ. 2005;33:1374.

4. Lawrence VA, Hilsenbeck SG, Noveck H, Poses RM, Carson لl. Medical complications and outcomes after hip fracture repair. Arch Intern Med. 2002:162:2053-7.

5. Vestergaard P, Rejnmark L, Mosekilde L. Increased mortality in patients with a hip fracture - effect of pre-morbid conditions and post-fracture complications. Osteoporos Int. 2007;18:1583-93.

6. Li L, Liu C, Yang J, Wu H, Wen T, Wang W, et al. Early postoperative controlling nutritional status (CONUT) score is associated with complication III-V after hepatectomy in hepatocellular carcinoma: a retrospective cohort study of 1,334 patients. Sci Rep. 2018:8:13406.

7. Aldebeyan S, Nooh A, Aoude A, Weber MH, Jarvey EJ. Hypoalbuminaemia-a marker of malnutrition and predictor of postoperative complications and mortality after hip fractures. Injury. 2017:48:436-40. 
8. Detsky AS, McLaughlin JR, Baker JP, Johnston N, Whittaker S, Mendelson RA, et al. What is subjective global assessment of nutritional status? J Parenter Enter Nutr. 1987;11:8-13.

9. Ignacio de Ulíbarri J, González-Madroño A, de NGP V, González P, González B, Mancha A, et al. CONUT: a tool for controlling nutritional status. First validation in a hospital population. Nutr Hosp. 2005;20:38-5.

10. González-Madroño A, Mancha A, Rodriguez FJ, Culebras J, de Ulibarri Jl. Confirming the validity of the CONUT system for early detection and monitoring of clinical undernutrition: comparison with two logistic regression models developed using SGA as the gold standard. Nutr Hosp. 2012;27:564-71.

11. Takagi K, Domagala P, Polak WG, Buettner S, ljzermans JNM. The controlling nutritional status score and postoperative complication risk in gastrointestinal and hepatopancreatobiliary surgical oncology: a systematic review and meta-analysis. Ann Nutr Metab. 2019;74:303-12.

12. Charlson ME, Pompei $P$, Ales KL, MacKenzie CR. A new method of classifying prognostic comorbidity in longitudinal studies: development and validation. J Chronic Dis. 1987;40:373-83.

13. Dindo D, Demartines N, Clavien PA. Classification of surgical complications: a new proposal with evaluation in a cohort of 6336 patients and results of a survey. Ann Surg. 2004;240:205-13.

14. Kanda Y. Investigation of the freely available easy-to-use software 'EZR' for medical statistics. Bone Marrow Transplant. 2013;48:452-8.

15. Nishi I, Seo Y, Hamada-Harimura Y, Sato K, Sai S, Yamamoto M, et al. Nutritional screening based on the controlling nutritional status (CONUT) score at the time of admission is useful for long-term prognostic prediction in patients with heart failure requiring hospitalization. Heart Vessel. 2017;32: 1337-49.

16. Kotera A. Geriatric nutritional risk index and controlling nutritional status score can predict postoperative 180-day mortality in hip fracture surgeries. JA Clin Rep. 2019;5:62.

17. Bohl DD, Shen MR, Hannon CP, Fillingham YA, Darrith B, Della Valle CJ. Serum albumin predicts survival and postoperative course following surgery for geriatric hip fracture. J Bone Joint Surg Am. 2017;99:2110-8.

18. Ryan S, Politzer C, Fletcher A, Bolognesi M, Seyler T. Preoperative hypoalbuminemia predicts poor short-term outcomes for hip fracture surgery. Orthopedics. 2018;41:e789-96.

19. Adogwa $O$, Martin JR, Huang K, Verla T, Fatemi $P$, Thompson $P$, et al. Preoperative serum albumin level as a predictor of postoperative complication after spine fusion. Spine. 2014;39:1513-9.

20. O'Daly BJ, Walsh JC, Quinlan JF, Falk GA, Stapleton R, Quinlan WR, et al. Serum albumin and total lymphocyte count as predictors of outcome in hip fractures. Clin Nutr. 2010;29:89-93.

21. Åhman R, Siverhall PF, Snygg J, Fredrikson M, Enlund G, Björnström K, et al. Determinants of mortality after hip fracture surgery in Sweden: a registrybased retrospective cohort study. Sci Rep. 2018;8:15695.

22. Cher EWL, Allen JC, Howe TS, Koh JSB. Comorbidity as the dominant predictor of mortality after hip fracture surgeries. Osteoporos Int. 2019;30: 2477-83.

23. Sankar A, Johnson SR, Beattie WS, Tait G, Wijeysundera DN. Reliability of the American Society of Anesthesiologists physical status scale in clinical practice. Br J Anaesth. 2014;113:424-32.

24. Rubenstein LZ, Harker JO, Salvà A, Guigoz Y, Vellas B. Screening for undernutrition in geriatric practice: developing the short-form mininutritional assessment (MNA-SF). J Gerontol A Biol Sci Med Sci. 2001;56: M366-72.

25. Ferguson M, Capra S, Bauer J, Banks M. Development of a valid and reliable malnutrition screening tool for adult acute hospital patients. Nutrition. 1999; 15:458-64.

26. Chiu HC, Chen CM, Su T-Y, Chen CH, Hsieh HM, Hsieh CP, et al. Dementia predicted one-year mortality for patients with first hip fracture: a population-based study. Bone Joint J. 2018;100-B:1220-6.

27. Steenson J, Vivanti A, Isenring E. Inter-rater reliability of the subjective global assessment: a systematic literature review. Nutrition. 2013;29: 350-2.

28. Bell JJ, Bauer JD, Capra S, Pulle RC. Quick and easy is not without cost implications of poorly performing nutrition screening tools in hip fracture. J Am Geriatr Soc. 2014;62:237-43.

29. Ritter SRF, Cardoso AF, Lins MMP, Zoccoli TLV, Freitas MPD, Camargos EF. Underdiagnosis of delirium in the elderly in acute care hospital settings: lessons not learned. Psychogeriatrics. 2018;18:268-75.
30. Espaulella J, Guyer H, Diaz-Escriu F, Mellado-Navas JA, Castells M, Pladevall M. Nutritional supplementation of elderly hip fracture patients. A randomized, double-blind, placebo-controlled trial. Age Ageing. 2000;29: 425-31.

31. Jie B, Jiang ZM, Nolan MT, Zhu SN, Yu K, Kondrup J. Impact of preoperative nutritional support on clinical outcome in abdominal surgical patients at nutritional risk. Nutrition. 2012;28:1022-7.

\section{Publisher's Note}

Springer Nature remains neutral with regard to jurisdictional claims in published maps and institutional affiliations.
Ready to submit your research? Choose BMC and benefit from:

- fast, convenient online submission

- thorough peer review by experienced researchers in your field

- rapid publication on acceptance

- support for research data, including large and complex data types

- gold Open Access which fosters wider collaboration and increased citations

- maximum visibility for your research: over $100 \mathrm{M}$ website views per year

At BMC, research is always in progress.

Learn more biomedcentral.com/submissions 